Meta

Journal des traducteurs

Translators' Journal

\title{
Approche socioterminologique des discours du nucléaire
}

\section{Valérie Delavigne}

Volume 40, numéro 2, juin 1995

Usages sociaux des termes : théories et terrains

URI : https://id.erudit.org/iderudit/002506ar

DOI : https://doi.org/10.7202/002506ar

Aller au sommaire du numéro

Éditeur(s)

Les Presses de l'Université de Montréal

ISSN

0026-0452 (imprimé)

1492-1421 (numérique)

Découvrir la revue

Citer cet article

Delavigne, V. (1995). Approche socioterminologique des discours du nucléaire. Meta, 40(2), 308-319. https://doi.org/10.7202/002506ar

\section{Résumé de l'article}

Une enquête de type socioterminologique, menée auprès du public et de spécialistes du nucléaire, montre que le vocabulaire de l'atome est générateur d'incertitudes et entaché d'inquiétudes sous-jacentes, et ce, malgré les efforts des responsables pour " mieux " communiquer. On peut cependant se demander si les discours trop lénifiants des dirigeants apportent de réelles réponses aux interrogations du public. 


\title{
APPROCHE SOCIOTERMINOLOGIQUE DES DISCOURS DU NUCLÉAIRE
}

VALIERII: DH:LAVICINE:

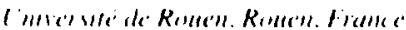

\begin{abstract}
Résumé

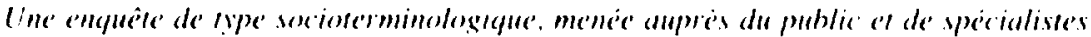

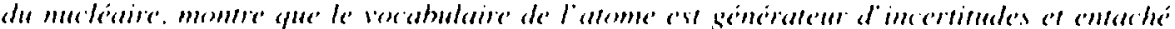

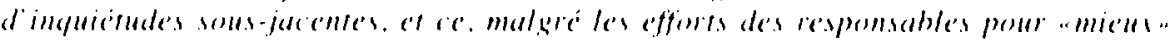

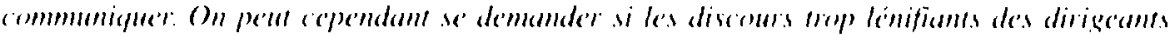

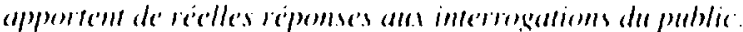

\section{( ADRE THEORIQI E EI METHODOI. KITUTE.}

La socioterminologie s'est constituée en réponse à une demande sociale el aux interrogations posées par le développement des sciences et techniques (Gambier 1987: Gaudin 199.3 a et $199.3 \mathrm{~h}$ ). Elle ne peut plus se limiter aujourd hui at la critique des outils conceptuels de la terminologic issue de lécole de Wuister, mais se doit de "passer à l"action". Il s"agit désormais de vérilier le caractere opératoire tant des concepts qu elle a élaborés que des méthodes qu elle a préconisées. Ine élude sur le vociabulatire du nucléaire proposé au grand public est l'ocicasion d'en tester l'opérativité.

l.a soxioterminologie s'occupe de termes et, par là. ne peut faire l'économie de l'usage' qui en est fatit. Son objet d'étude ést donc constitué par lat réalité des discours spécialisés dans lesquels s insèrent les termes, mis en auve au cours d’interactions spécifiques. Les lermes circulent en effet dans des sphères d activités multiples el multiformes, véritables "nouds" interdisciplinaires. par le hiais des discours. Elle aftinne sal volonté de description. a linstar de la sociolinguistique. L a prise en compte des discours et leur analyse permettent. en replaçant les termes au sein des pratiques langagières et sociales concrètes, de comprendre leurs fonctionnements et leurs civentuels dystonctionnements.

Il y a donc déplacement de l'ohjet d'étude par rapport à l'approche terminologique traditionnelle, dans la mesure où ce sont les discours spécialisés dans leur globalité qui deviennent objet d’intérêt. et plus seulement les termes. L intérêt est porté sur les formes in situ, et non sur les unités isolées, sorties de leur contexte de production, figées dans une définition. entités immuables. hors de tout enjeu social. Les termes sont, dans notre optique, considérés comme les éléments constitutifís d activités sociales variées.

I a diversité des situations de communication spécialisées commence à être prise en comple par les linguistes et apporte de nouvelles connaissances sur le fonctionnement des lextes écrits de spéciallé (Jacobi 1994: 78-79). Mais l'oral reste peu étudié. Face à ce manque, la sociolerminologie, outre son intérêt pour l'ensemble des écrits émis dans une sphère d'activiké, préconise des procédures d'enquêtes de terrain. C'est ce à quoi nous nous sommes altachéc.

\section{IE. VOCABLIAIRE DU : NUCIEAIRE.}

La recherche que nous rapportons ici porte sur la question de la diffusion el de l'impact d'un technolecté spécificue: le vocabulaire du nucléatire, et de l'appropriation de

Har 17.2 ows 
ce “lexique spécialisé»' par des locuteurs «non spécialistes». La problématique que nous engageons nous mène donc entre terminologie et lexicologie. C"est en effet dans une optique de déspécialisation de termes que notre contribution trouve sa place. Cependant, si ale discours de VS |Vulgarisation scientifique| ne constitue pas un corpus pour le repérage des termes", il n'en reste pas moins, ainsi que le souligne Marie-Françoise Mortureux (1994: 64). qu'il constitue un type de discours spécifique au sein de l'ensemble des discours spécialisés et que des termes y figurent.

Loin de limiter notre objet d'étude, s intéresser à la diffusion du vocabulaire du nucléaire. à sa réception et à sa reformulation par un public a priori non prévenu des concepts mis en discours. élargit le champ de recherche. En effet, il s'agit non seulement de $s$ "informer des pratiques discursives à l'ceuvre chez les locuteurs ordinairement dénommés "spécialistes», mais également des productions langagières émises par les médias. les organismes-relais entre l'information issue des responsables de la production de l'énergic nucléaire et le public "non spécialiste". Ce public "non spécialiste" peut tout aussi hien être un public constitué d'autres types de "spécialistes". Yves (iambier avait déjà montré au sujet des pluies acides (1987) ce que Louis (iuespin appela ediscours d'interface" (1989) mıs en évidence notamment avec le vocabulaire des biotechnologies. II s'agit donc de prendre en compte la diversité des situations de communication, sachant que le public $n^{\circ}$ a pas accès a une source unique. mais à des discours multiples et parfois contradictoires.

\section{Corpus}

L'analyse terminologique est précédée d'un travail terminographique de recueil des données. La démarche socioterminographique nécessite de collecter les termes dans leur contexle discursif, oral ou écrit.

\section{Corpus ćcrit: la question du domaine}

La nature de notre travail donne une orientation particulière au recueil des données écrites dans la mesure où notre objet est composé de textes publiés ou diffusés pour un destinataire spécifique: le grand public. Notre premier critère de sélection est donc celui d'un public présumé. Ce critère nous place dans la problématique des discours de vulgarisation, avec d'éventuelles réserves². Nous avons limité dans le temps le recueil d'écrits à l'après-Tchernobyl (mai 1986). présupposant que cet accident technologique majeur constitue une coupure dans la représentation que le public peut se faire de l'énergie nucléaire. Au niveau géographique. nous nous sommes restreinte à des textes français. présupposant que les sensibilités nationales en face de ce type d'énergie étaient susceptibles de variations.

Voila pour les critères externes. Le critère interne de sélection est l'apparition dans le titre. les interlitres ou le corps des textes, de termes faisant explicitement référence à l'exploitation nucléaire civile. Ont donc été rassemblés des discours qui circulent autour de la radioactivité : production d'électricité, applications médicales, industrielles, ou à des fins de recherche...

Cependant, malgré cette restriction à l'utilisation du nucléaire à des usages civils. nous avons affaire à un "domaine» polymorphe où interviennent aussi bien des notions de physique et de chimie nucléaire que de droit. de médecine. de géologie. de météorologie, etc. Par exemple, si l'on s'intéresse spécificuement à l'exploitation de l'atome pour la production électrique, les activités et, par là. les discours qui tournent autour du syntagme ćnergie macléaire sont pluriels et variés. À la base du vocabulaire employé. il y a une technique: la fission nucléaire. La sécurité nucléaire en est un des indispensahles corollaires, avec pour objectif la protection non seulement des hommes, travailleurs du nucléaire ou population locale. mais aussi de la faune et de la flore. Vont donc être pris en compte 
pour la construction d'un établissement nucléaire, la résistance des matériaux ou l'écoulement des eaux, pour ne retenir que deux aspects bien éloignés du travail d un spécialiste de la ratdioprotection. par exemple, yui aura pour sa part à se soucier du respect des réglementations par le personnel.

De fait. comme la montré Yves Gambier au sujet des pluies acides:

lne science, une technique réfère à d'autres sciences, dautres techniques - elles-mêmes hranchées sur d'atutres. |... | il n'y a pas de cdomaine" sans adomainese connexes: un "domaine" revient ators à un noud de connexions. (Gambier $1991: 37$ )

Ce dernier critire de sélection montre l'aspect non opératoire de la notion extralinguistique de "domaine" telle que l'envisagent les terminologues dans la lignée d'Eugen Wüster. Les textes que nous avons recueillis sont le reflet de lat diversité de cette sphère d'activité qu'on ne peut clore de façon ferme et définitive.

\section{Corpus oral}

Par ailleurs, une enquête orale menée sous forme d entretiens semi-directifs a permis d'évaluer la connaissance el la compréhension que le public interrogé a du vocabulaire du nucléaire ${ }^{3}$ ainsi que la façon dont les spécialistes de la radioactivité en appréhendent la vulgarisation.

Nous nous proposons done de monerer quelques aspects du fonctionnement de la terminologie du nucléaire. Le détour par l'histoire de la discipline ne peut manquer détre source denseignement pour comprendre la façon dont les termes sont refus par les locuteurs non spécialistes. Comme le souligne Roger Goffin (1989) 95), on ne peut analyser la terminologie de l'attome sans s'attarder sur l'atventure du nucléaire qui l'a constituée.

\section{Aperçu historique}

La terminologie du nucléaire est relativement récente : la découverte de la radioactivité naturelle $n$ 'a pass encore cent ans (elle date de 1896). Lat terminologie de l'atome. dès sa diffusion hors du cercle des scientifiques, a hénélicié d'un engouement spectaculaire: tout ce qui écait radioactif scmblait bénéfique.

Le terme rediecactif. né dans le monde de la physique. a connu une diffusion rapide en dehors du cercle des scientifiques par le hiais d'applications médicales. La curiéthérapié. destinée a soigner le cancer, contribue à montrer laction positive des rayonnements radioactifs, tandis que, pour soigner tels ou tels maux. on avalait des pilules radioactives ou l'on se plongeait en cure dans des eaux radioactives. Si l'on commence a prendre conscience dans les années vingt qu une trop forte radioactivité proveque des troubles pathologiques. on continue à penser que de petifes doses non seulement n'ont pas d'incidence négattive sur la santé, mais, au contraire, procurent un effet stimulant. Marie Curie, elle-même. préconise dans son Tratté de radionatrivité, publié en 1934, l'année de sat mort. l'ingestion d'eau raddioactive, l'inhalation d'air contenant du radon et des injections de substances radioactives comme pratiques the rapeutiques (cite par (ioldschmidt 1987: 55).

le déclenchement de la Seconde (iuerre mondiale et la course a la bombe qu clle at entrainée ont suscité une atmosphère de secret qui a perduré au-dela du lancement du programme de consuruction des centrales nucléaires françatises dans les années 1970. Des mouvements antinucléaires se sont alors emparés des termes. $A$ la polémique suscitée par l'accident de Tchernohyl el au traumatisme qu il constitue. les politiques répondent par la nécessité de la transparence. transparence qui s'institutionnalise tardivement floi de décembre 1991). Elle se traduit par la "mise en tourismè des sites nucléaires: ceux-ci sont ouverts au public: y sont organisées conférences, visites guidées. expositions qui doivent répondre aux questions du public, tandis que de la documentation est à sa disposition. Les 
brochures mettent en scène la terminologie de l'atome en tentant d'effacer les attitudes dysphoriques qu'elle est susceptible d’entraîner (Delavigne 1994).

l.es représentations restent cependant marquées par l’image de la bombe et de l"accident: les enquêtés ont pratiquement tous évoyué Hiroshima et Nagasaki : et le nom de Tchernobyl. récurrent dans les discours des interviewés, réveille les inquiétudes latentes du public. Depuis l'effondrement du régime soviétique. la connaissance de l'état des centrales nucléaires de l'Est suscite de vives inquiétudes. Des trafics de substances radioactives sont mis au grand jour. Löinquiétude du public tend à se focaliser sur les sites de l'Europe de l'Est et influe sur les représentations: par contraste. la technologie occidentale paraît très fiable.

\section{Un peu de morphologie}

S intéresser à la dimension cognitive des termes telle qu elle peut être perçue par le public nécessite un détour par la morphologie. En effet, devant un terme au signifié obscur. les locuteurs ont tendance à considérer le signifiant afīn de tenter de le rapprocher d'un autre terme connu et d'en dégager ainsi du sens. Or, examiner rapidement les structures morphologiques des lermes les plus fréquents dans les discours destinés au public permel de mettre en évidence des signifiants que l'on peut qualifier de relativement "transparents». Bien entendu, le degré de technicité des unités varie en fonction des textes el de leur finalité.

Tentons une rapide description morphologique des termes présents dans les textes destinés au public. Deux types de termes apparaissent: les uns ont trait à la recherche fondamentale. les autres à sa mise en application. Nous ne constituons pas là deux "domaines" distincts, mais il se trouve que le fil diachronique joue sur la formation des unités en présence.

Un certain nombre d'unités issues de la langue généralet : moyau, parficulé, activité. rayommements viennent dénommer les nouveaux concepts. Les unités de mesure sonı désignées par des éponymes (Röngen. Becque'rel. ('wrie) tandis que les transuraniens. éléments chimiques que mettent en évidence les chercheurs. sont formés à partir de noms de planètes: aramium, néprunium, plufomium, puis de noms de lieux géographiques el de physiciens célèbres (américium. curium. coslifornium. cinstcinium).

Lorsque l'on passe aux applications de ces découvertes. on retrouve également un grand nombre de termes simples. empruntés à la langue courante et à d'autres langues spécialisées. qui se voient dotés d'un sémantisme neuf comme divergencé. pile (remplacé

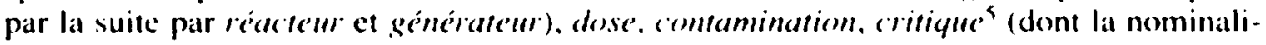
sation entraîne la création vers 1965 du néologisme ('riticite'). colis, ('mballage', hlindage'.

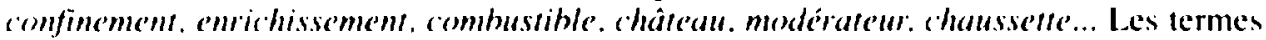
complexes" voient leur fréquence augmentée. Deux types de termes complexes apparaissent: les uns possèdent des joncteurs tandis que les autres n en possèdent pas?.

Notre corpus de termes est composé pour une large majorité d"un type d'unités qu'Émile Benveniste désignait par «synapsie». Il ne semble pas que le fait soit surprenant. vu qu'il considérait que ce procédé de formation était "appelé à une productivité indéfinie" (1974: 172). Notre corpus suit donc une tendance confirmée de la langue scientificue (cf. par exemple. le vocabulaire des biotechnologies dans Assal 1992). Un grand nombre d'unités terminologiques sont des syntagmes avec joncteurs. La majorité de ces termes complexes sont formés sur la structure nom + prép. + nom. comme par exemple

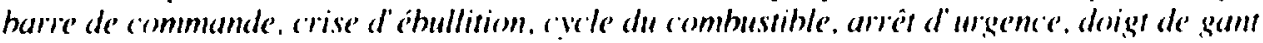
ou nom + prép. + déterminant + nom comme conduite d'un réacte'ur. Ces structures peuvent se combiner pour former des modèles du type : nom + prép. + (nom + adj.)

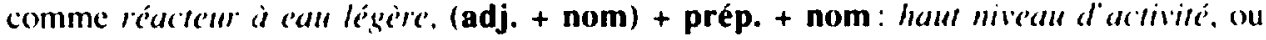


nom + prép. + déterminant + (nom + adj.) comme démantilement d'unc contrale muclécure' ou piriode d'un cil'ment radioartif.

D'autres composés n'ont pas de ligament synaptique. On trouve quelquess syntagmes asyndétiques. c'est-ì-dire d'unités terminologiques formées sur le modèle nom + nom.

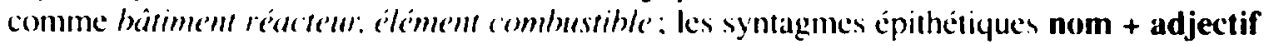

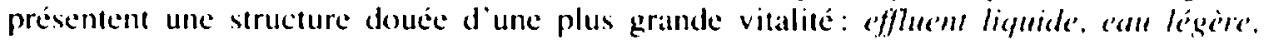

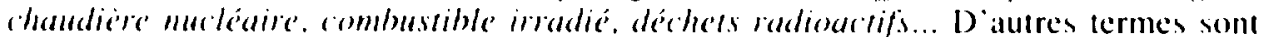
composes sur ke modele (nom + adjectif) + adjectif comme desc maximale admissible. mais aussi sur la structure nom + sigle comme combustible MOX. filicere RLP.

Selon Roger (ioffin (1989: 97), la siglaison est intervenue tres tôt dams le vocabulaire du nucléaire pour des ratisons de protection des recherches, lat volonté de secret influençant les comportements langagiers. ("est ainsi par exemple que le premier réacteur

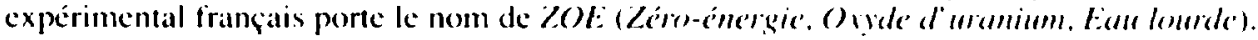
Cette tendance à la siglaison sest muée en tradition prégnante: Ies carractéristiques des

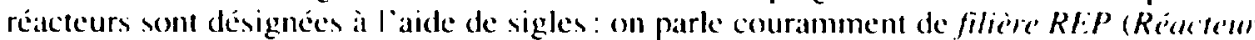

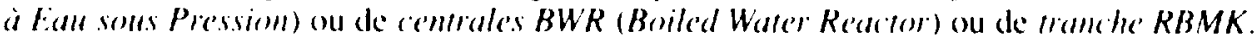
Il est à noter que les sigles synonymes anglais et français $(R L P$; PWR) apparaissent régulièrement en concurrence dans les textes à destination du public, ce qui peut générer quelyue confusion.

Lés sites nucléaires sont désignés par le nom de leur site d’implantation (Marcoule. Paluel. Buge's, (irancline's...). parfois atu grand dan des autochtones qui refusent de voir le

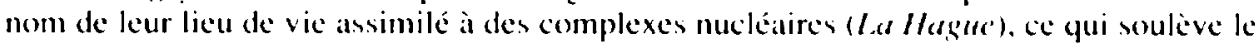
probleme de lat perception de cetle industrie (voir siupra).

Dams lensemble, une remarque s impose: ce rapide survol morphologique des termes présents dans les textes en direction du public montre qu à l'exclusion des sigles qui opacifient la communication vers le public, la leminologie du nucleaire n'offre pas alu non-spécialliste des "monstres", it leimage de certains vocabulatires techniques comme ceux que l'on trouve dans les biotechnologies, par exemple (hibridation sommaticue par fusion

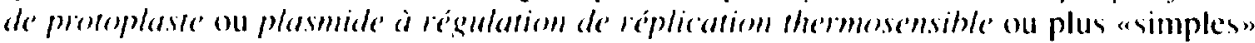

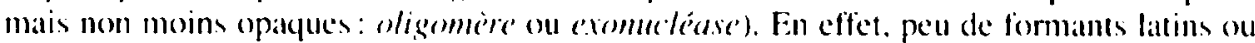
grees interviennent ditns la formation des termes qui circulent dans les textes émis en direction du public, hormis ceux qui comprennent les préfixess radir-. thermo-. electron-

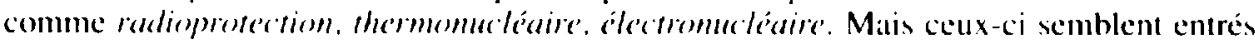
dans la langue commune, même si l'ateception de lélément redie- est parfois légèrement différente (ke formant ent rattache à radielegie'). Nous avons donc affaire à une terminologie que nous pourrions qualifier de relativement motivée: le sémantisme des termes est fréquemment révélé par des marques formelles. Un grand nombre de mots de la langue générale interviemment dan ce vocabulaire, soit en tant qu unités isolées, soit combinés.

\section{FNTRE CI.ARTE: FT INC 'OMPREHENSION}

Sont-ils clairs pour autant pour le public? D’une enquête menée dans la région de Paluel, commune française qui abrite une centrale nucléaire. il ressorl que l'on doive répondre par la négative (Delavigne et Guespin 1992). Faut-il accuser la tradition de secret qui a eu cours depuis la Seconde (juerre mondiale ou conclure à une mavaise qualite de l'information issue des responsables en direction du public"? Sans rementre en ciatuse les efforts des respomsibles pour une tramsparence accrue, it faut remarquer que d'autres facteurs peuvent jouer. 


\section{Une dimension sacrée}

Une métaphore anthropomorphique apparaît de façon récurrente dans ce vocabulaire: les établissements nucléaires sont dotés non seulement d'un crortu. mais aussi d'un bouclier. L'uranium 238 est fertile, c'est-à-dire qu'il peut subir une fission, et a une demivic de 4.47 10$)^{\prime \prime}$ ans, ce qui signifie que la radieactivité de l'uranium diminue de moitié au cours de celte durée (ce calque de l'anglais, demi-vié, est proserit par les arrêtés de terminologie au profit de période, mais reste très utilisé à loral). Ce vocabulaire révèle la dimension quasi sacrée d'une conquête technologique qui "constitue un véritable défi à la nature dont elle transgresse les interdits» (Zonnabend 1989). Celle époxpe porte d'ailleurs un nom: liere du macliaire.

Lévolution de celte lechnologie moderne a fait franchir à l'homme la frontière entre sacré et profane. ce qui se traduit sur le plan du langage. Le terme mème de transmutation (modification d'un élément chimique en un atome nouveau) n'est pas sans évoquer quelyue sorcellerie. une alchimie érrange. Les savants ont pénétré les secrets de la matière: le nucléaire défie la nature en brisant le cour même des atomes. Ce n'est pas la simple propos d'humeur. $\mathrm{N}^{\prime} \mathbf{a}-\mathrm{t}-\mathrm{on}$ pas parlé a propos des centrales nucléaires des cathédrale's du $X X^{\prime \prime}$ siècle'? Si l'on délaisse les termes évocateurs en eux-mèmes de cette dimension sacrée. on peut remarquer que tous les sites nucléaires sont conçus selon une subdivision en zomes (zome publicure. surveillée, contrôlée) accessibles seulement à une certaine catégorie de personnes habilitées à pénétrer ces différentes zones. L'analogie avec les édifices religieux est frappante. Il nous semble que cet aspect n est pas à négliger. dans la mesure où il donne à cette terminologie une connotation qui $n$ 'est peut-être pas sans rapport avec la "peur du nucléaire”, rejoignant par là les angoisses engendrées par le progrès technique, à l'instar par exemple des manipulations génétiques.

\section{Les termes en discours : les discours des responsables}

Comment ces termes sont-ils convoqués dans les textes émis par les responsables? Nous nous sommes altachée à scruter ces textes atin d identifier les stratégies discursives mises en cauvre. Examinons rapidement un extrait d'une brochure mise à la disposition du public dans une centrale nucléaire et publiée par Électricité de France: ('éntrales muclécuires el emirommement (EDF 1990).

Nous avons conscience que cette documentation reste dans lensemble peu lue et que ce n'est pas le moyen d'accès aux informations que privilégie le public. C'est en effet généralement à partir de sources plus accessibles que le public s'approprie les dennées sur le nucléaire. Les connaissances quant à l'utilisation de la radioactivité et aux risques qui lui sont liés proviennent souvent de films aux scénarios catastrophistes: la culture filmique joue un rôle non négligeable dans la construction de l'image publique du nucléaire. Subsistent parfois quelques souvenirs scolaires. C'est plus généralement par le biais des médias, par la relation d'incidents el d'accidents spectaculaires comme Tchernohyl, par des reportages que sont acquis les savoirs sur le nucléaire (Delavigne et Guespin 1992).

Néanmoins. examiner les énoncés des responsables du nucléaire revient à prendre en compte un type de discours que l'on peut qualifier de "primaire». (e sont en effet ces discours qui fonctionnent comme discours-sources et qui seront ensuite repris par les médias. Dans la pluralité des énoncés-origines. il nous semble indispensable d'examiner quels sont les termes présents puisque ce sont eux qui réapparaîtront dans les discours profaness et d'examiner au moyen de quelles stratégies ils sont convoqués dans les énoncés des producteurs.

Ces: brochures sont publiées par les grandes entreprises du nucléare el destinées à un public qui, a priori, ne connaît ni les concepts ni les principes à la base de la production d'énergie nucléaire. In certain nombre de stratégies révèlent l'adaptation des émetteurs 
au destinataire visé : emploi de procédures descriptives qui exhibent un travail de reformulation, importance des énoncés métalinguistiques et usage autonymique des termes. Cés brochures sont médiation entre les experts et le public. Elles constituent, dans l'ensemble des énoncés émis sur l'énergie nucléaire, un discours légitime.

Les alccidents nucleaires possibles en cours dexploitation ont fait l'objel d'éludes approfondies. Touter les hypothèses ont été envisagees: defatlance materielle, erreur humainke. scisme. chute d'avion... Dans tous les cas, des dispositions sont prevues pour limiter les effets sur l'environnement à un niveau très faible, sans consequences pour la population.

léchec complet de toutes les mesures de sécurite en le percement de la cuve par la fusion du ceur ont même éle imaginces : lenceinte de conlinement est prévue pour supporter la pression de la vapeur yui sechapperait alors du circuit primaire et la radionativite resterail confince dans lenceinte.

Depuis 1986 , une précaution supplémentaire a été prise : pour permettre d'évacuer une partic des ga/ vers lextéricur en cas de pression excessive. toutes les centrales sont progressivement equipes de filtres à sable: ceux-ci permettratient de retenir $90 \%$ des proxluits radioactils relaches, el de preserver l'integrite de l'enceinte de continement.

Enfin. dam le cas hatulement improbable oi un accident xe produirait, deux plans

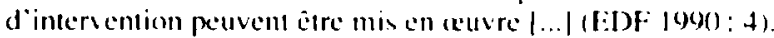

Un certain nombre de termes apparaissent dans ce court extrait : aceident muclécuire.

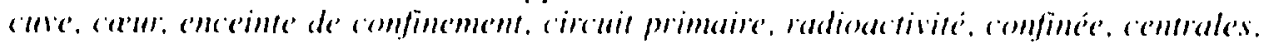

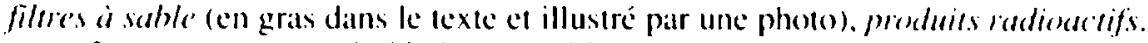

les syntagmes relatifi à un accident sont contrebalancés par des cooceurrents apaisatlts:

\begin{tabular}{|c|c|}
\hline ACCIIDENT & COOCCURRENTS \\
\hline accidents nuc|éaires possibles & objes d'études approfondies \\
\hline toutes les hypotheses & onl élé envisiagées \\
\hline dans lous lescas & $\begin{array}{l}\text { des dispositions sont prevues } \\
\text { limiter les effets sur l'environnement } \\
\text { sams conséquences pour la population }\end{array}$ \\
\hline $\begin{array}{l}\text { lechec complet de touten les mesures de } \\
\text { sécurite et le percement de lat vapeur } \\
\text { par fusion du cour }\end{array}$ & ont mềne ćte imagines \\
\hline la pression de la vapeur qui a echapperait & $\begin{array}{l}\text { Penceinte de confinement est prévue } \\
\text { pour supporter }\end{array}$ \\
\hline lat raclionativite & resterait confinese dams l'enceinte \\
\hline pression excensive & une précalution supplémentaire a été prise \\
\hline $90 \mathrm{c} / \mathrm{d}$ des produits radoactifis relaches & retenir \\
\hline l'intégrilé de l'enceinte de conlinement & prescrver \\
\hline cass où un accidemt se produirait & hatutement improbable \\
\hline
\end{tabular}


Les désignations des origines d'un ahypothétique accident" sont citées: "défaillance matérielle, erreur humaine, séisme, chute d'avion...", mais l'accident en lui-même n'est envisagé que sous un aspect unique : “fusion du ccrur». Aucun autre moyen discursif n'est proposé pour se le représenter: l'accident reste abstrait, inimaginable - impossible - he répondant pas aux interrogations du public (ef. supra) qui risque. en conséquence de s'arrêter sur des idées erronćes.

Le radical comfin- (comfinement, comfince) apparait trois fois et permet d'envisager. par contraste, la conséquence de cet ahypothétique accident s: la diffusion de substances radioactives. La notion de "confinement" est centrale en sûreté nucléaire. Le lecteur peut donc appréhender le fait que l'émission de radioactivité est le danger à éviter à tout prix.

le discours est construit vers une euphorisation. Soulignons la valeur d'insistance des signes employes: :études approfondies". "toutes les hypothèses ont été envisagées"s. "dans toms les ras, des dispositions sont prévues", "l'échec complet de toutes les mesures de sécurité $|. .$.$| ont mìme été imaginées". "une précaution supplémémaire a été prise",$ "dans le cas hauteme'nt improbahle où un accident se produirait". Less termes sont pris dans un système lénifiant, sans neutralité.

Cependant à ce discours officiel viennent se greffer, au cours des entretiens menés avec des cadres travaillant dans l'industric nucléaire, des énoncés moins péremptoires. Si la compétence des travailleurs du nucléaire n'est pas remise en cause, il n en reste pas moins que la négation du risque est moins catégorique. Lorsqu est demandé à un spécialiste du nucléaire non plus de s'exprimer sur sa pratique professionnelle, mais de donner son opimion sur l'utilisation de l'énergie nucléaire et ses dangers potentiels, moult précatutions oratoires ainsi que de plus nombreux ratages apparaissent dans le fil de l'énoncé, tandis que le paralinguistique (débit de la voix, intonations. pauses...) révèle un discours moins construit et plus malaiséx

\section{Le problème de la réception}

Comment les locuteurs non spécialistes reçoivent-ils les termes du nucléaire? De l'enquête menée auprès du public ressort une insécurité linguistique face à la terminologie de l'atome : celle-ci est certes connue dans ses grandes lignes, mais est sujette à des distorsions conceptuelles. Il faut admeltre avec François Gaudin qu “identifier n'est pas comprendre" (1993a: 152). Pour que les mots fassent sens, encore faut-il comprendre la valeur qu'ils prennent au sein du système linguistique. Les entretiens montrent que kes locuteurs chargent sémantiquement les termes à partir de leur signifiant en se référant à d'autres usages, mais une "culture nucléaire" insuffisante fait attribuer à ces formes des signifiés parfois bien éloignés du signifié original.

\section{Un accidem inconcerable}

Quels signes sont convoqués lorsqu'un accident mucléaire est envisagé par les locuteurs interrogés" L'image qui apparaît contraste avec l'euphorisation des discours des responsables. Nous voici pourtant en présence d'un terme à l'apparente clarté et qui. cependant, est le siegge d'interrogations pressantes de la part du public.

Au niveau des vocables employés par les locuteurs pour considérer l'occurrence d'un accident, l'improbable est désigné par des mots sémantiquement très ouverts: «s'il y avait le moindre probleme'». "admette" qu'il arrive un tric comme ca", "au cas ou il y aurait

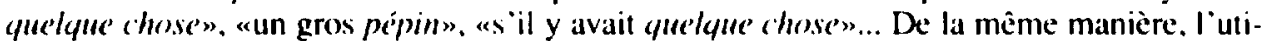
lisation du pronom démonstratif $\varsigma a$ associé au conditionnel permet d'évocquer l'accident sans le nommer vraiment.

Un accident est généralement envisagé par des non-spécialistes sous la forme d'une explosion. malgré les dires des responsables selon lesquels cela est physiquement 
impossible. Les images convoquées sont violentes: "quand elle explose la centrale". "Ça semballe en l'air", "ça fuse partout ef lu es touché partout". "si elle se met à exploser, on est cuit tout de suite». «moi je n’ai pas peur que ça saute». En fait l'image prégnante de la bombe n'est sans doute pas sans conséquence sur la perception violente que les locutcurs se font de laccident. Notons aussi l'apparition dans plusicurs entretiens du syntagme abif

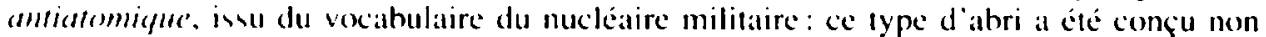
pour parer à l'éventualité d'un aceident nucléaire, mais à celle d'une attaque atomique. Il révele, parmi d'autres symptomes. l'amalgame de l'usage de l'atome à des fins civiles el militaires.

En niant toute possibilité d'accident. en vidant le terme de tout contenu, les responsables ne répondent pass aux représentations que le public se fait d'un accident nucléaire. Notons que les locuteurs n envisagent généralement l'éventualité d'un tel accident qu après un premier discours confiant: l'inquićtude est d'abord niéce avant d'apparaitre en cours. voire en fin d'entretien.

\section{I.a radiosucinits;}

Examinons maintenant rapidement la façon dont un des concepts centraux de l'industrie de l'atome est perçu: la radionarivité. Le terme est connu de l'ensemble des locuteurs interrogés. La radioactivité ne connaît pas de défïnition simple : phénomènè non perceptible. éprouvable par aucun des cing sens, mesurable uniquement par des appareils, cetle notion est rattachéc à des phénomènes connus. comme un "gaz" ou une "effervesence», Raccrocher la radioactivité à des schémas cognitifs plus habituels permet én somme d'apprivoiser le concept.

Il es en fait beatucoup plus facile de parler asur" la radioactivité que ade» la ractioactivité. Dans les extraits des entretiens menés avec des personnes extérieures au nucléaire, la radioalctivite n’apparaît pas spontanément comme un phénomène natturel. Blle est essentiellement perşue comme quelque chose d'artificiel. Des commentaires sur le danger que la radioactivité représente sobtiennent aisément. Le terme suscite des associations du type: "ce"st vicieux", "ben la radioaclivité, cest une image pour moi pas tellement positive". "la radioactivité. c"ent de l'air polluć par radiation". Mais expliquer en quoi consiste ce "phénomène" apparait comme une entreprise extrêmement malatséc, voire impossible.

La notion de pollution domine : la radioactivité est spontanément associéce à un danger inclassable, insidieux, qui "va partout". le terme est a l'origine d'inquiétudes el associé à des mots comme "cancers", "leucémies", "malformations généliques", «monstres".. II nest fait mention yue d'une radioatctivité trop importante.

Si l'on examine les détinitions écrites par des spécialistes, on se rend compte yue la notion est loin d"êre simple. ("est à la fois la propricté d'un élément démettre des rayonnements et celle émission elle-même: "propriété de cerrains atomes de se désintégrer. d'émettre des rayonnements", "propriélé d'émettre des particules et des rayonnements", "cimission d'un rayonnement accompagné de la transmutation de lélément qui rayonne". "désintégration de nombreux noyaux". Toutes ces définitions extraites de documents destincis au public ne somt pas nécessairement très éclairantes.

le fait qu elle me soit perceptible par aucun des cinc sens en fait un phénomène insaisissable, difficilement classable, yui ne correspond à aucun des dangers connus habi-

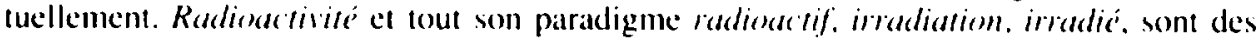
termes abstraits, difficiles à appréhender et que l'on ne peut rattacher à alucun emploi concret. La racine commune a ces termes, radio- (rayrom), reste dans celle acception difficile à catégoriser: la notion sous-jacente de arayonnement ne repose sur aucune évidence sensible: on ne peut la rapporter au quotidien. Ce signifié sans chair pour les locuteurs interroges lait du nucléaire une énergie difficilement conceptualisable. d une grande abstraction. Or, 
moins une unité est claire, moins elle est reformulable; c'est ce qui explique que les énoncés obtenus au cours de notre enquête quant au terme radicractivité soient essentiellement de nature épilinguistique. Chez certains locuteurs, la relation entre la notion de radioartivitó et celle de rayonnement est totalement absente.

Ce début d'enquête permet donc de mettre en évidence la manière dont le public appréhende les mots du nucléaire et comment il $s^{\circ}$ y heurte. Peut-être permettra-1-elle de ce fait d'engendrer des solutions et de répondre ainsi à la vocation glottonomique de la socioterminologie.

\section{PISTES POUR UNE INFORMATION RÉUSSIF:}

La diffusion des connaissances est souvent évoxuée en termes de traduction “intralinguale» d'une langue de spécialité à la langue commune, el est liée aux pratiques de reformulation. Communiquer avec des profanes nécessite en effet de négocier le sens des termes utilisés, de trouver un langage commun si l'on veut que l'interaction soit réussie.

Nous avons noté plus haut que de nombreuses unités simples ou des éléments qui entrent dans la composition de termes complexes sont des emprunts à la langue commune. L'artivité d'un élément, par exemple, a quelque chose à voir avec le prototype d'une activité quelle qu'elle soit. Chaque terme ainsi emprunté ne saurait être complètement coupé de la langue générale. Il ne s'agit pas d'homonymes: une même isotopie unit chaque usage. 11 pourrait être judicieux pour une information réussie de mettre les différents usages en relation afin de mettre en place une sorte de réseau cognitif.

Pour pallier les problèmes liés à la notion abstraite de «rayonnement". la métaphore peul être un outil incontestablement heurislique dans la mesure où elle permet l'ancrage du connu sur du non-connu. La métaphorisation n'est pas en terminologie un procédé d'ornement stylistique, mais cest à insérer dans la large problématique du rapport entre l'activité cognitive et l'activité discursive qui permet sa réalisation" (Assal 1992: 178). Elle est une manière de penser el de concevoir: sa finalité est conceptuelle.

C'est un moyen de rattacher des notions abstraites et peu évidentes à des schèmes intellectuels déjà présents, de tisser des liens entre savoirs ancien el nouveau. En s’ancrant sur des connaissances présentes, les notions difficiles peuvent s'intégrer dans les systèmes de représentations préexistants. C'est d'ailleurs une pratique spontanée des locuteurs quand ils tentent d'expliquer des concepts qu'ils connaissent mal. Ils assimilent la radioactivité à un "gaz» ou à une "effervescence». Ces métaphores permettent d'appréhender l"inconnu en se rétérant à une expérience personnelle qui foumit un support imaginatif, de créer une certaine familiarisation avec le signifiant qui permet d'avoir accès, par petites touches, au signifié.

La figure fait naître du sens. Rapprocher la radioactivité de phénomènes du même type. comme le rayonnement lumineux par exemple. doit faciliter l'accès à des savoirs peu évidents. De la même manière que la science en train de se faire «trouve ses mots» par un mouvement métaphorique qui fertilise l'appareil conceptuel (voir à ce sujet Gaudin 1993: Stengers et Schlanger 1988), le processus peut être inversé en matière de vulgarisation. La fertilisation métaphorique peut donc se faire dans un double mouvement: certains mots de la langue générale créent l'intuition découvrante, puis, après coup, la langue de spécialité vient informer la langue générale. augmentée d’un sens nouveau pendant son séjour au pays des spécialistes. La pluralité des sens doit donc permettre de faire circuler les lermes dans les deux sens.

\section{CONCLUSION}

Le $X X^{*}$ siècle nucléaire a vu naître trois révolutions scientifiques. La physique de la relativité est une pure abstraction, hors "culture"; elle ne touche pas le grand public et 
reste confinée dans le cercle des spécialistes. Les progrès de la biologie, pour leur part. ont engendré le mythe des manipulations génétiques en touchant aux secrets de la vie. La troisième révolution. la révolution nucléaire, a fait naitre un autre mythe : celui de la destruction totale ou. en tout cass, de la destruction de l'intégrité humaine. L'image de monstres engendrés par une calastrophe nucléaire d'origine civile ou militaire reste présente. Jointe à elle. l'idé d'une souillure universelle se lit jusque dans les termes techniques: comlamination. "mot qu 'on aimerait bien faire disparaître, mais on n'y arrive pass". Liangoisse suscitéc par le nucléaire, généralement refoulée. s'exprime par une parole malaisée qui va au-dela de la difficulté des concepts en jeu: les enquêtes menées en lémoignent. On peut voir dans cet embarrass à dire la trace d'un certain désarroi. Est-ce une des raisons qui poussent les responsables a proposer un discours lénifiant et consensuel"? C"est une hypothèse.

\section{Notes}

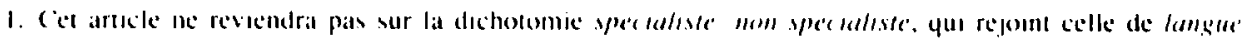

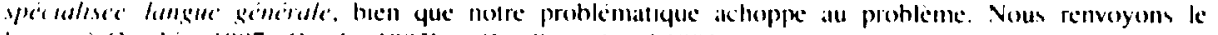

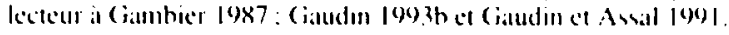

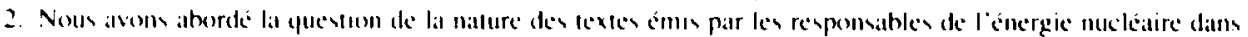
Delavigne (19)4.

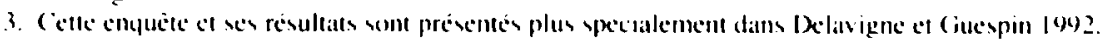

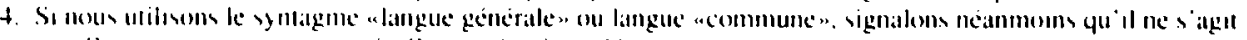

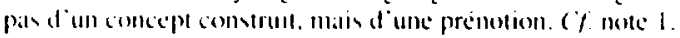

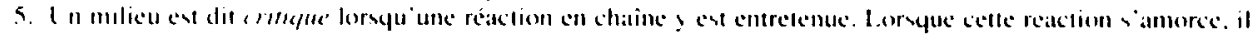

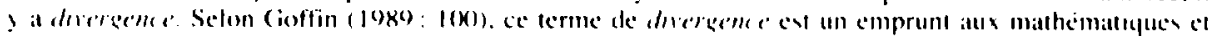
devignce déux courtes yuu s'scartemt.

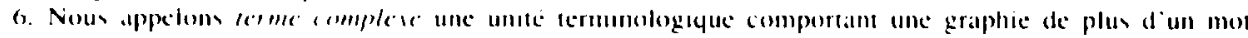
Ypegraphlopus.

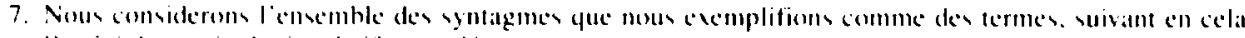
l'arrific ake lemmologic relat if au nucleaure

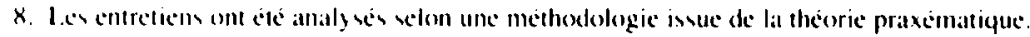

4. Extrant diun entretien mente avec un spécialiste de l'énergete nucléate.

\section{REFERENCES}

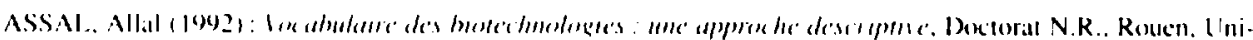
crilci de Rollen. $317 \mathrm{p}$.

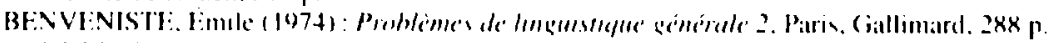

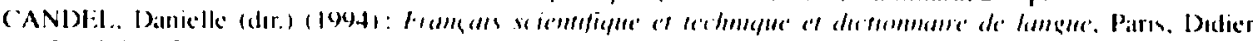
Firudition, $201 \mathrm{p}$

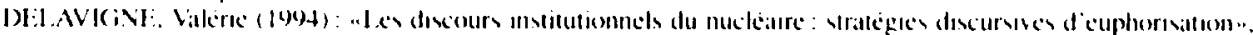

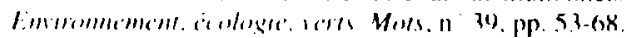

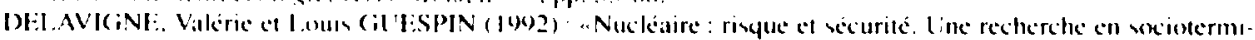

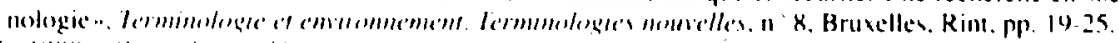

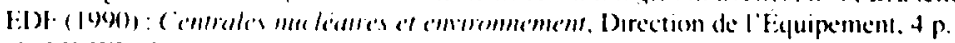

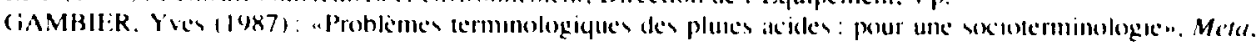

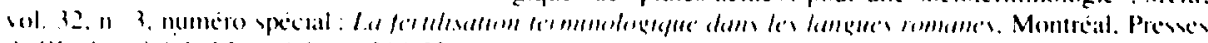
de I'l niscrute de Mommial. pp. 314-120.

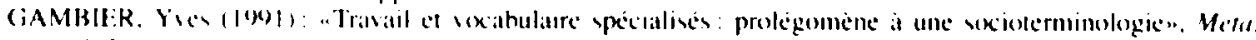

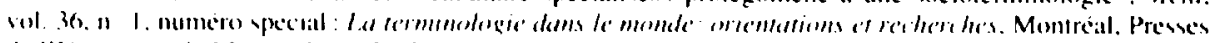
de l'l inversite de Montrial. pp. 31-.5x

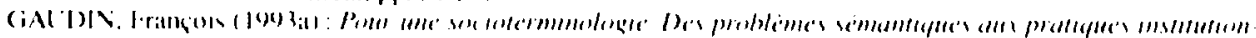

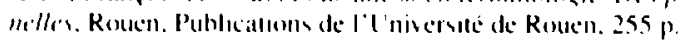

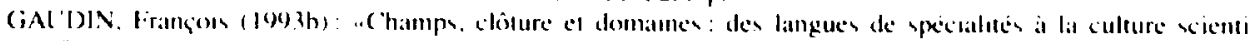

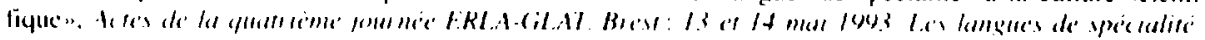

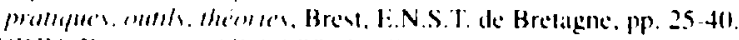

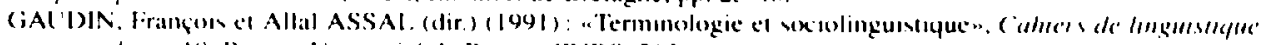
weiale. $n$ ix. Rouen. I noversits de Rouen. (NRS. $213 \mathrm{p}$ 
(iOFFIN. Roger (1989): “al a terminologic des sciences el techniques nucléaires, un cas de diachronie recente”.

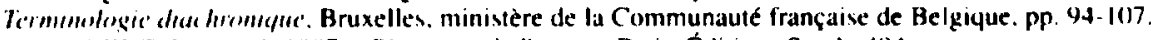

(i()LIDSCHMIDT, Bertrand (1987): Pismmern de latonte. Paris, Éditions Stock. 484 p.

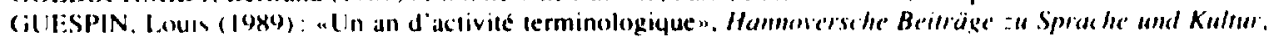
n'1, Iniversite d'Hanovre. pp. $70-77$.

JAC (OBI. Danel ( 19941 : "I exique et reformulation intradiscursive dans les documents de vulgarisation scienti-

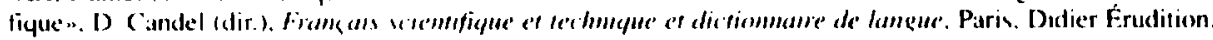
pp. 77.91 .

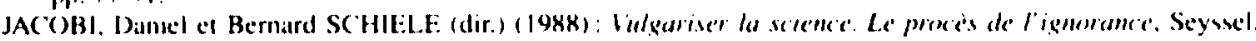
Editioms ( 'hamp Vallon. $286 \mathrm{p}$

MORTIIRFIX. Marie-Franģoise $(198 K)$ : "La vulgarisation scientifique. Parole médianc ou dédoublée". Jacobi

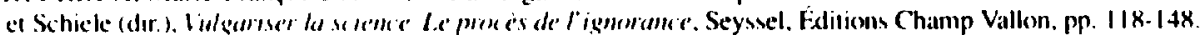

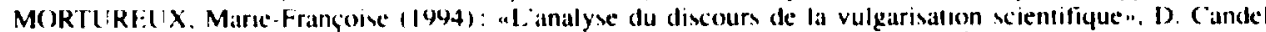

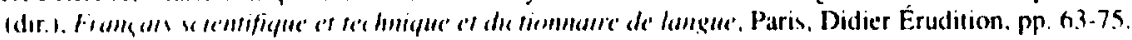

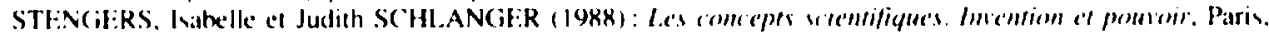
l.al dicouvertí. $13.5 \mathrm{p}$

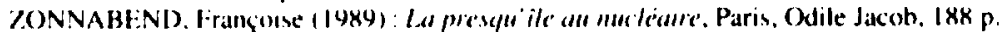

